\title{
Thermal modulator array for analyte modulation and comprehensive two-dimensional gas chromatography
}

Ben V. Burger, Tertia Snyman, Wina J. G. Burger

Laboratory for Ecological Chemistry, University of Stellenbosch, Private Bag X1, Matieland 7602, South Africa

Willie F. van Rooyen

Central Electronic Services, University of Stellenbosch, Private Bag X1, Matieland 7602, South Africa

\begin{abstract}
Segmented thermal modulators were fabricated for the modulation of column effluent in onedimensional gas chromatography, comprehensive two-dimensional gas chromatography, and as sample introduction devices in other analytical techniques. Thermal desorption of analytes from the modulator was achieved by computer-controlled application of large DC currents at low voltages sequentially to consecutive segments or stages of the modulator heaters for periods between 100 and $400 \mathrm{~ms}$ per segment. This procedure produced sharply focussed analyte peaks. Unsegmented heaters and heaters having $2,4,6,8$, and 10 segments or stages were compared with respect to their focusing efficiency. The 10-stage heater was found to give sharper focusing than heaters with a smaller number of segments. Analyte pulses with peak widths at half-height of ca. $150 \mathrm{~ms}$ were generated by the 10-stage heater. The application of a 10-stage modulator in a comprehensive twodimensional analysis of a light petroleum condensate gave results of acceptable quality.
\end{abstract}

Keywords: Modulator; Sample introduction; Comprehensive two-dimensional GC.

\section{Introduction}

The development of a new type of thermal modulator (sweeper) as an interface between the two columns in comprehensive two-dimensional gas chromatography [1], has given new impetus to research in this field and was followed by the introduction of other robust and efficient modulators [2-4]. Earlier modulators were made by coating capillaries with thin electrically conductive films [5, 6]. Their low thermal mass was, understandably, considered to be a desirable characteristic of these devices and coating the modulator capillaries with thicker layers of metal was thought to be a step in the wrong direction because the time required to cool down became excessive due to the thermal inertia of such systems [7].

In exploratory measurements, we have found that although this is in principle correct, the slower cooling rate of thin stainless-steel capillaries could be acceptable under certain operating conditions. The focusing effect achieved with the sweeper [1], on the other hand, was considered a very desirable feature of that instrumentation. Several possibilities to obtain focusing efficiencies approaching those obtained with the sweeper but without using moving parts in the column oven, were considered. Resistive heating of a tapered stainless-steel modulator heater, for example, was discarded in favor of analyte focusing by the rapid resistive heating of consecutive segments ("thermal scanning") of a thin stainless-steel capillary. The efficiency of a modulator heater of this type clearly depends on making electrical contact with the segments without creating cold spots at the points of contact. The construction of modulator heaters in which this prerequisite is satisfied, results of temperature calibrations, and the determination of optimum operating parameters will be presented in the present paper. 


\section{Experimental}

\subsection{Thermal modulator array}

Lengths of stainless-steel tubing (Sinox; $110 \mathrm{~mm} \times 520 \mu \mathrm{m}$ OD x $340 \mu \mathrm{m}$ ID as well as $110 \mathrm{~mm} \times 320$ $\mu \mathrm{m}$ OD $\times 160 \mu \mathrm{m}$ ID) were manufactured to exacting tolerances by Schoeller Werk GmbH (D-53940 Hellenthal, Germany). To make a 10-segment heater for $200 \mu \mathrm{m}$ ID modulator capillaries, one of the 520- $\mu \mathrm{m}$ stainless-steel tubes was cut into eleven 10-mm connectors, the tips of these connectors were hammered flat and silver-soldered (hard-soldered) to a 110-mm length of tubing at ca. 10$\mathrm{mm}$ intervals, starting $5 \mathrm{~mm}$ from one end of the $110-\mathrm{mm}$ tube as shown in Figure 1 . The tips of the connectors were flattened to prevent the molten solder from entering the tubular connectors and increasing their electrical conductivity. To restrict electrical conductivity at the joints, as little silver solder as possible was used and the connectors were soldered to the tube with their flat surfaces at right angles to the axis of the tube.

Silver solder filings with a mass of ca. $0.4 \mathrm{mg}$ were selected and melted in the presence of soldering flux with a micro oxygen/propane burner on an unglazed porcelain plate to produce small spheres. The stainless-steel tube and connectors were configured on the unglazed porcelain plate and carefully taped to the plate to keep the individual parts in place during the soldering process in which the silver solder spheres were used. With a little experience, the preparation of a 10-segment array took about an hour. Glass-fiber insulated copper leads $(0.85 \mathrm{~mm}$ diameter) were temporarily clamped together in the form of a flat belt and were permanently kept in this arrangement with polyimide, applied at suitable points, and cured in an oven at the appropriate temperature. The ends of the copper leads were bent and trimmed to correspond with the spacing of the short stainlesssteel connectors of the modulator heater array, and were silver-soldered to the free ends of the connectors. Thermal modulator arrays with smaller numbers of segments were made in a similar manner. Arrays for 100- $\mu \mathrm{m}$ ID modulator capillaries were made from the 320- $\mu \mathrm{m}$ OD stainless-steel capillaries.

\subsection{Modulator capillaries}

Fused-silica capillary tubing (200 ImID) (Polymicro Technologies, Phoenix AZ, USA) was deactivated and statically coated with the apolar phase PS-255 (DB-1 equivalent) (Petrarch Systems Inc., Bristol PA, USA) at a film thickness of $3 \mathrm{Im}$. Depending on the envisaged application, a suitable length of this coated capillary was removed and a $95-\mathrm{mm}$ section appropriately measured out to prepare a modulator capillary with an effective length of $95 \mathrm{~mm}$. Using a 10-IL syringe, an acetone/pentane mixture containing $30 \%$ pentane was very carefully pushed into one end of the capillary up to the first mark and left in that position for ca. 5 min., after which the solvent was withdrawn, and the residual solvent and solvent vapour removed with dry nitrogen introduced into the capillary from the opposite end. This procedure was repeated about 15 times until a drop of the solvent removed from the capillary did not leave a visible ring of stationary phase when it was allowed to evaporate from the surface of a mirror. The procedure was repeated from the other end of the capillary. The capillary that fits snugly into the heater was sprayed several times with a Teflon ${ }^{\mathrm{TM}}$ suspension to facilitate its removal from the heater if, for some reason, it had to be replaced. The modulator capillary was connected to the capillary column with a press-fit connector of the appropriate dimensions.

\subsection{Power supply}

An electrical sequencing system with which currents of between 1 and $20 \mathrm{~A}$ could be instantaneously and consecutively supplied to the segments of the modulator heaters, was built according to the diagram given in Figure 2. The system consists of a $5 \mathrm{~V}, 25 \mathrm{~A}$ power supply, a current limiting and a segment switching circuit, the output of which is switched to any one or more of the segments of the segmented modulator heater. The system is controlled by an internal 
microprocessor that, once the operating parameters have been downloaded into it from an external computer via a RS232 link, operates independently. The external computer can then be used for data acquisition, for example. The programmable parameters are: current ( 1 to $20 \mathrm{~A}$ in steps of $1 \mathrm{~A}$ ), number of segments (1 to 20), and time duration of current pulses (10 to $2500 \mathrm{~ms}$ per segment). The pause time can be selected by either programming the required pause time, or by programming the pause time for an imaginary additional segment without actually connecting a segment to the corresponding output terminals on the sequencer. The system has been designed with an internal microprocessor, so that a dedicated computer is not required once the operating parameters have been set. The system is further controlled with only 'Start' and 'Stop' buttons.

\subsection{Temperature calibration}

Linear thermocouples were made by but-connecting Chromel and Alumel wires ( $0.25 \mathrm{~mm}$ diameter). The thermocouples were rolled between carborundum paper sheets (3M-314, grade 360 ) supported on two smoothly planed wooden blocks until they reached diameters of $170 \mathrm{Im}$ and $80 \mathrm{Im}$, suitable for insertion into fused-silica capillaries with inside diameters of 200 and $100 \mu \mathrm{m}$, respectively. To calibrate a heater array a thermocouple with the appropriate diameter was inserted into an uncoated fused-silica capillary which was in its turn inserted into the heater array and could be shifted back and forth inside the stainless-steel capillary to monitor the thermal behavior of the segments and soldered junctions of the modulator heaters under working conditions.

The copper leads to the current sequencer were fed through one of the GC-MS interface ports of the GC. The modulator array was installed in a position facing the fan of the GC, i. e. in the position it would normally be used for column effluent modulation or comprehensive two-dimensional gas chromatography, and was held in this position with a clamp outside the oven. The output of the thermocouple was recorded on a strip-chart recorder.

\subsection{Modulation}

All modulation experiments were done on a Carlo Erba Mega 5300 gas chromatograph configured with a split/ splitless sample inlet and FID. The modulator capillary was inserted into a heater of the corresponding inside diameter in such a way that the coated section of the modulator capillary was positioned centrally between the first and last connectors on the heater. A 20-m $\times 0.25-\mathrm{mm}$ fusedsilica column coated with $0.25 \mu \mathrm{m}$ of PS-089 (DB-5 equivalent) at a film thickness of $0.25 \mu \mathrm{m}$ was installed in the GC and was connected to the modulator capillary with a press-fit connector. The other end of the modulator capillary was connected to the FID in the prescribed manner and the copper leads connected in the correct sequence to the current sequencer. Installation as described here usually takes less then 20 minutes.

\subsection{Data acquisition}

Data produced in comprehensive two-dimensional gas chromatographic analyses were acquired using Borwin integration software (JMBS Developments, 38600 Fontaine, France) and the data were visualized with PyramidTM software written for this purpose by Dr Barrie Hopkins (Mass Spec, Johannesburg, South Africa).

\section{Results and discussion}

The interest in modulator technology over the last decade centers largely around the modulation of the effluent of the first column in comprehensive two-dimensional gas chromatography. However, thermal modulators can also be used for other purposes. Devices for the enrichment and pulsed release of volatile organic compounds for gas chromatographic separation or for direct detection by a detector, or mass spectrometer, are essentially thermal modulators. Such devices can also be used 
to generate sharp gas chromatographic effluent pulses for electro antennographic detection in insect pheromone research. In planning the present project, the goal was to develop a thermal modulator that would be suitable for a variety of applications, would allow extended unattended operation, and which could be constructed at low cost from readily available materials by any reasonably well equipped workshop.

It was argued that the rapid consecutive heating of the segments of a modulator heater containing the modulator capillary, a technique that could perhaps be described as thermal scanning of the modulator capillary, could be expected to produce analyte pulse widths approaching those obtained with the sweeper. For various reasons such as, for example, the risk of working with high voltages in the oven, resistive heating with high currents was preferred. The temperature generated in the segments by the application of currents of several amperes is such that the modulator will burn out if the current is left switched to a segment for longer periods. However, by selecting the appropriate combination of the magnitude of the current and the duration of the energizing pulse, rapid heating without any danger of burn-out could be achieved (vide infra). No modulator burn-out has occurred during the three years we have been using this system.

The creation of cold spots at the positions where the copper leads from the power supply are attached to the heater was avoided by making electrical contact via short connectors of the stainless-steel capillary of which the heater itself is made. This resulted in the contact being made via connectors that are themselves resistively heated. An absolute minimum of silver solder was used to avoid colder spots at the junctions due to the good electrical conductivity of the silver solder. Internal temperature measurements showed no appreciable heat transfer between segments when energizing pulses shorter than $500 \mathrm{~ms}$ are used. The sectors of a 10-segment heater could typically be operated at between $1 \mathrm{~A}$ at $0.4 \mathrm{~V}$ and $20 \mathrm{~A}$ at $1.6 \mathrm{~V}$, although the required temperature increments were normally obtained at currents lower than $10 \mathrm{~A}$.

An additional advantage of using a heater array instead of a heater consisting of only one or two segments is that a specific segment is only heated for the short time it takes the desorbed volatiles to be swept from that segment, whereas the entire length of an unsegmented heater has to be heated for the duration of the desorption. The stationary phase is thus not subjected to so much thermal stress in the segmented array. Perhaps for this reason practically no stationary phase bleed was observed in analyses of mineral oil samples up to C32.

Experiments were done with various sequencer parameters at different isothermal oven temperatures and oven temperature programs. It was found that temperature increments generated by different electrical currents were independent of the oven temperature or programming rate. The information such as that given in Figure 3 for a 10-stage modulator can therefore be used to select combinations of parameters that would give any appropriate temperature increment. It has to be taken into consideration that the information in Figure 3 is only applicable to work in GCs of the same type or model, i. e. in which the air is stirred at the same speed. Other temperature calibration graphs or tables have to be compiled if a modulator is installed in another model GC. However, since the temperature increment is independent of the oven temperature, it will only be necessary to do a calibration for one set of combinations of pulse times, for example from 100 to $400 \mathrm{~ms}$ in steps of $100 \mathrm{~ms}$, and current values that will give the temperature increment required by the envisaged application.

Examples of the results of experiments done with the 200- $\mu \mathrm{m}$ ID modulator capillary are given in Figure 4. The temperature profiles in Figure 4.A, obtained at an isothermal oven temperature of $150^{\circ} / \mathrm{min}$ and energizing pulses of $200 \mathrm{~ms} / \mathrm{segment}$, show that although the heater did not cool right down to the oven temperature between current pulses, the temperature increments are large and rapid, and that the modulator temperature returned to within a few degrees above the oven temperature between energizing pulses. The temperature profiles in Figure 4.B were obtained at an oven programming rate of $4^{\circ} / \mathrm{min}$. However, as can be expected, the segments did not cool down 
sufficiently to trap volatiles eluting from a capillary column or other sample-introducing device when very rapid heating of the segments without any pause time was employed (Figure 4.C). Similar results were obtained with the 100- $\mu \mathrm{m}$ ID modulator. An example of the modulation of pentacosane in a 10-stage modulator is given in Figure 5. Analyte pulse widths in the order of 145 ms were obtained using a $20-\mathrm{m}$ column programmed at $2 \% \mathrm{~min}$. Sharply modulated pulses were obtained for alkanes from $\mathrm{C}_{4}$ to $\mathrm{C}_{32}$ at programming rates lower than $4^{\circ} \mathrm{C}$. Because no provision was made for auxiliary cooling of the modulator, breakthrough was observed at higher programming rates. The contour plot of a comprehensive two-dimensional analysis of a light petroleum condensate is shown in Figure 6.

\section{Concluding remarks}

A combination of simple design, low cost, uncomplicated installation in any gas chromatograph without having to make any changes to the instrument, and reliability in unattended operation, makes the thermal modulator described here an attractive alternative to other modulators that have been used for column effluent and sample introduction in gas chromatography, mass spectrometry, and comprehensive two-dimensional gas chromatography.

As far as pulse widths are concerned, it is clear that the modulator in its present version and with a modulator capillary of $0.20 \mathrm{~mm} I D$ cannot compete with existing systems, some of which give pulse widths of less than $10 \mathrm{~ms}$ [4]. However, it will produce excellent results in applications that do not require the narrowest possible pulse widths. 
Figures

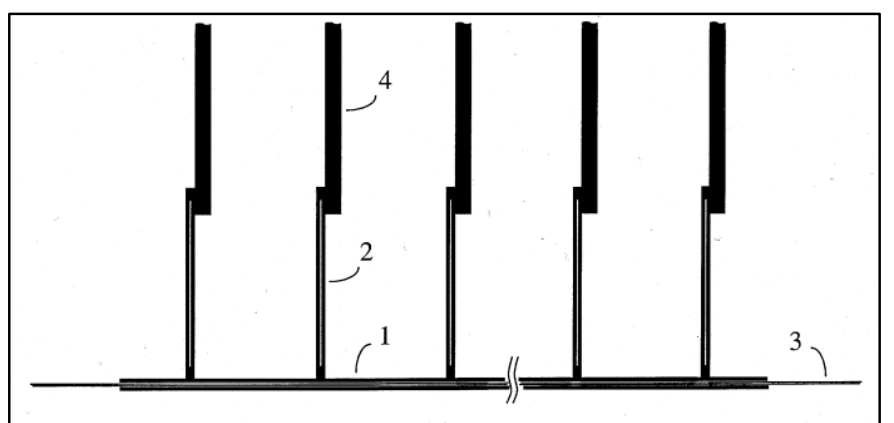

Figure 1. Schematic diagram showing four segments of a multi-stage thermal modulator array. 1. Stainless-steel capillary. 2. Stainless-steel connectors with flattened tips, made from the same stainless-steel capillary tubing. 3. Fused-silica modulator capillary. 4. Copper leads to the power supply.

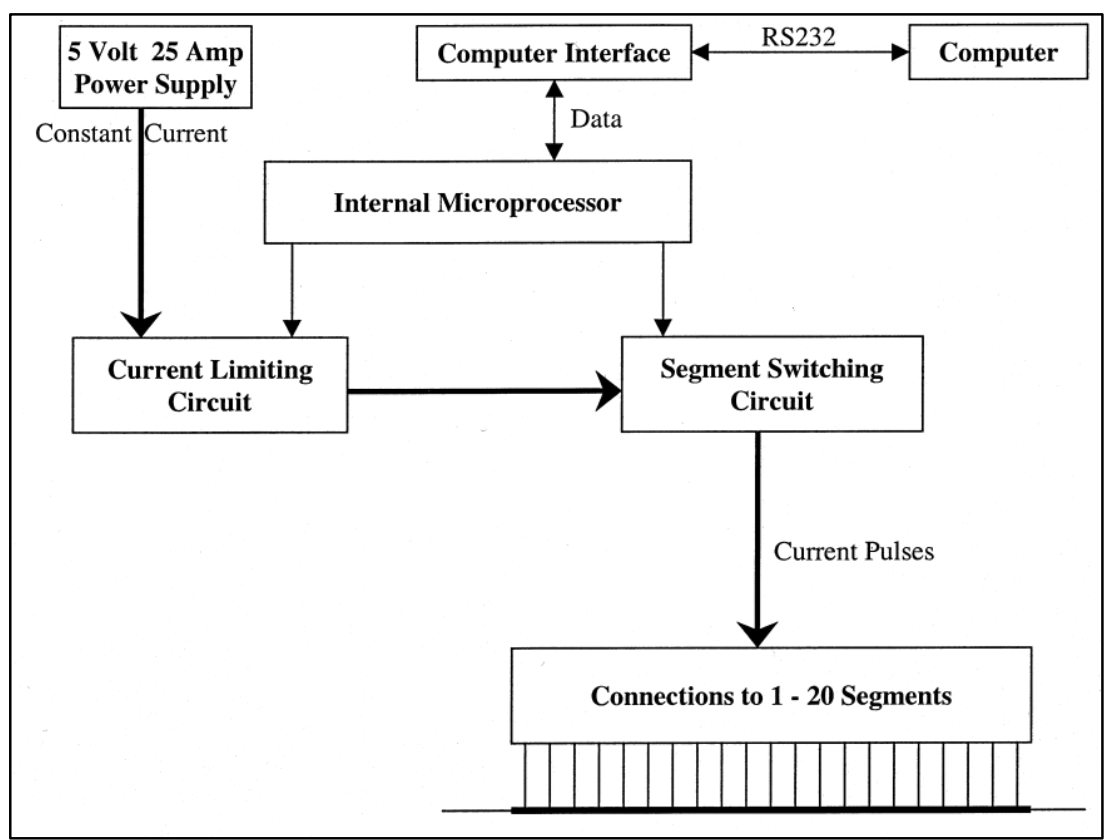

Figure 2. Schematic diagram of the power supply (current sequencer).

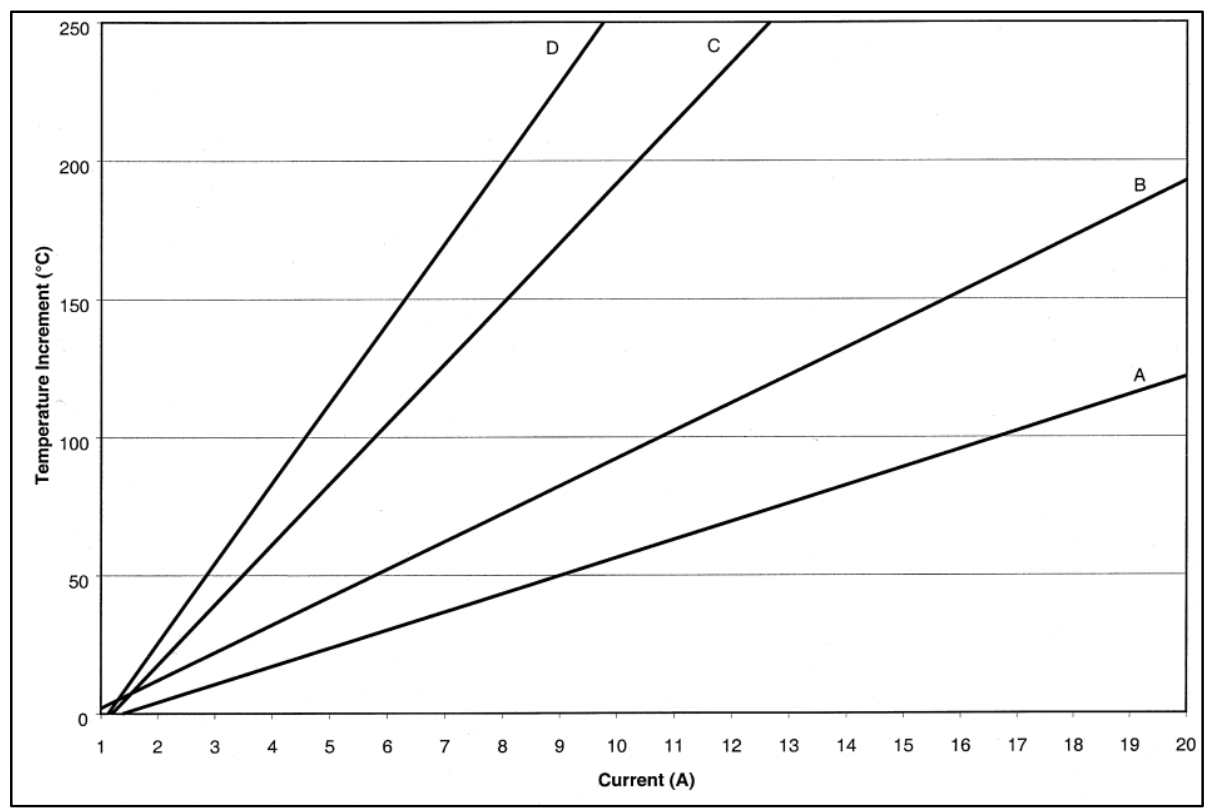

Figure 3. Plots of temperature increment $\left({ }^{\circ} \mathrm{C}\right)$ vs current flowing through one segment of a 10 -stage modulator. Pulse duration: $A=100 ; B=200 ; C=400 ; D=600$ ms/segment. 


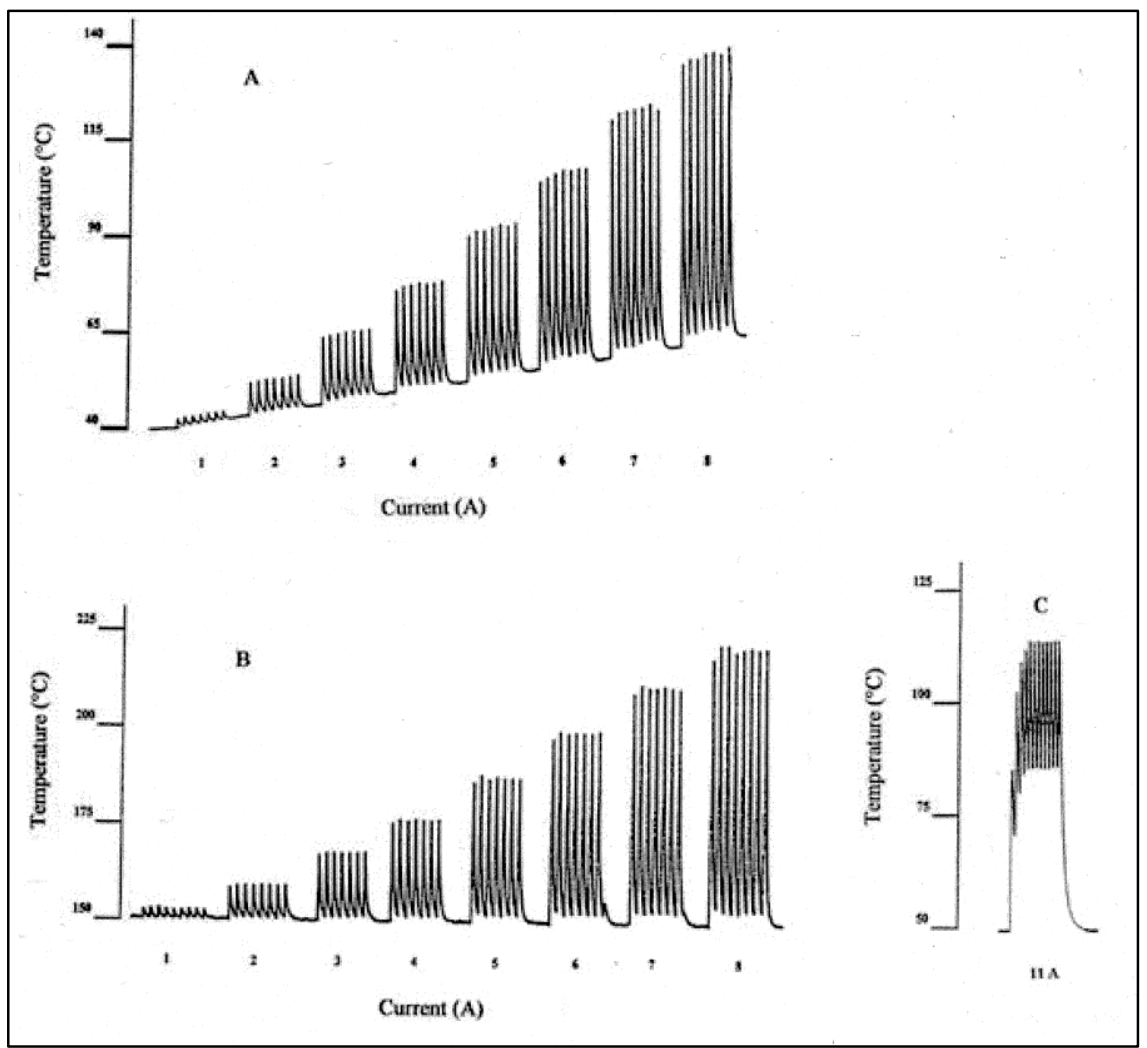

Figure 4. Thermal behaviour of one segment of a segmented modulator array: $(A)$ isothermal $G C$ oven temperature $150^{\circ} \mathrm{C}$, electrical pulse 1 to $8 \mathrm{~A}$, duration $200 \mathrm{~ms} / \mathrm{segment}$, pause time $3 \mathrm{~s}$; (B) oven programmed at $4 \% \mathrm{~min}$ from $40^{\circ} \mathrm{C}$, electrical pulse 1 to $8 \mathrm{~A}$, duration $200 \mathrm{~ms} /$ segment, pause time $3 \mathrm{~s} ;(\mathrm{C})$ isothermal oven temperature $50^{\circ} \mathrm{C}$, electrical pulse $11 \mathrm{~A}$, duration $100 \mathrm{~ms} /$ segment, no pause time.

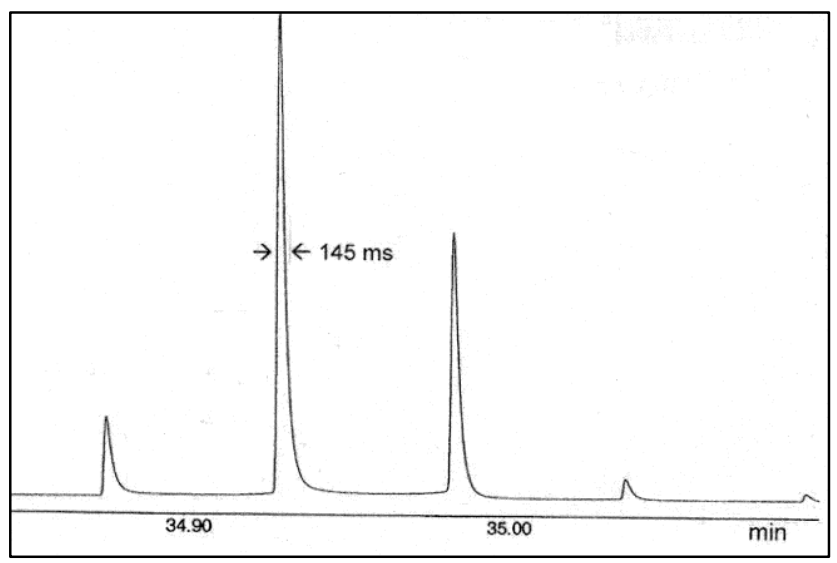

Figure 5. Modulation with a 10-stage modulator of pentacosane $(0.033 \mu \mathrm{g})$ eluted from a capillary column $(20$ $\mathrm{m} \times 0.25 \mathrm{~mm}$, coated with $0.25 \mu \mathrm{m}$ of polydimethylsiloxane and programmed at $2 \% \mathrm{~min})$. Modulator parameters: electrical pulse $9 \mathrm{~A}$, pulse duration $200 \mathrm{~ms} /$ segment, temperature increment $80^{\circ} \mathrm{C}$, pause time $1.3 \mathrm{~s}$. 


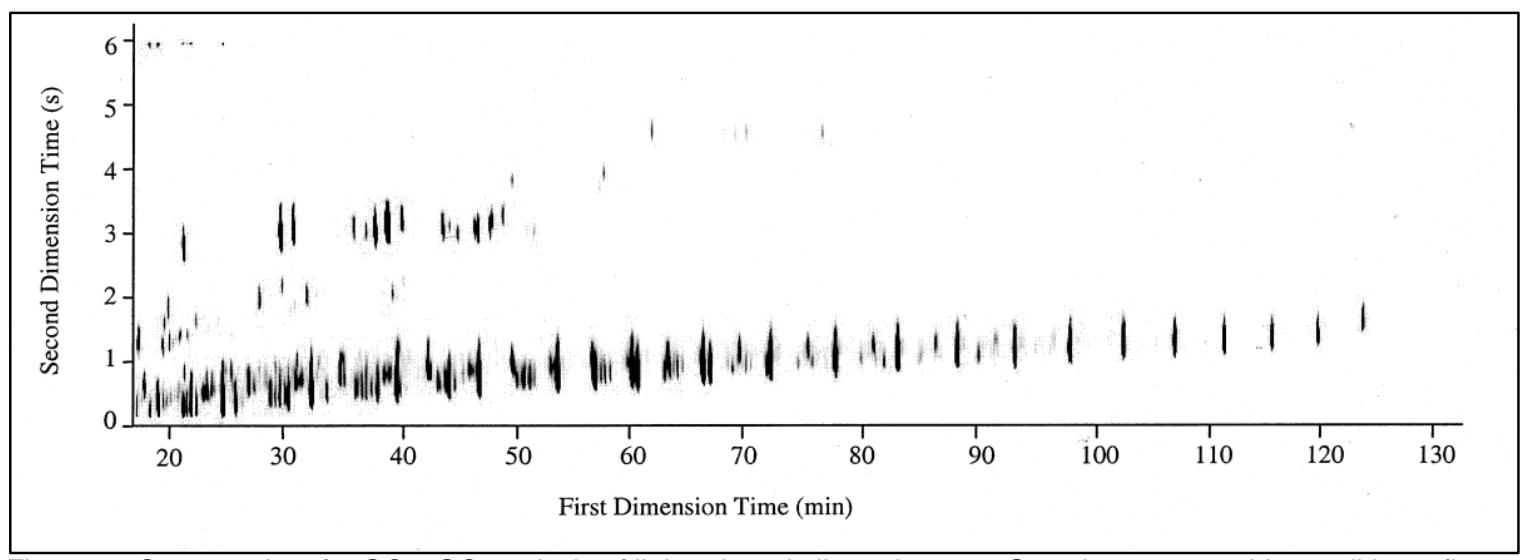

Figure 6. Contour plot of a GC $\times$ GC analysis of light mineral oil condensate. Gas chromatographic conditions: first column, $30 \mathrm{~m} \times 0.25 \mathrm{~mm}$ ID coated with $0.5 \mu \mathrm{m}$ PS-255; second column, $1 \mathrm{~m} \times 0.20 \mathrm{~mm}$ ID coated with $0.14 \mu \mathrm{m}$ $\mathrm{OV}-701$; mean $\mathrm{H}_{2}$ flow at $40^{\circ} \mathrm{C}, 64 \mathrm{~cm} / \mathrm{s}$. Modulator parameters: 10 -stage modulator pulsed at $300 \mathrm{~ms} / \mathrm{segment}$; temperature increment, $80^{\circ} \mathrm{C}$; pause time, $3 \mathrm{~s}$.

\section{Acknowledgements}

Support by the University of Stellenbosch and the National Research Foundation, Pretoria, of the research reported in this paper is gratefully acknowledged.

\section{References}

1. J.B. Phillips, R.B. Gaines, J. Blomberg, F.W.M. van der Wielen, J.M. Dimandja, V. Green, J. Granger, D. Patterson, L. Racovalis, H.J. de Geus, J. de Boer, P. Haglund, J. Lipsky, V. Sinha, E.B. Ledford, Jr., J. High Resol. Chromatogr. 1999, 22, 3-10.

2. R.M. Kinghorn, P.J. Marriott, J. High Resol. Chromatogr. 1999, 22, 235-238.

3. E.B. Ledford, Jr., C. Billesbach, J. High Resol. Chromatogr. 2000, 23, 202-204.

4. J. Beens, M. Adahchour, R.J.J. Vreuls, K. van Altena, U.A.Th. Brinkman, J. Chromatogr. A 2001, 919, 127-132.

5. J.B. Phillips, Z. Liu, U.S. Patent 5135 549, 1992.

6. J.B. Phillips, Z. Liu, U.S. Patent 5196 039, 1993.

7. W. Bertsch, J. High Resol. Chromatogr. 2000, 23, 167-181. 